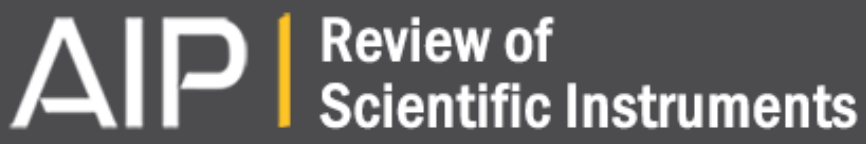

New apparatus for thermal diffusivity measurements of refractory solid materials by the periodic stationary method

J. Khedari, P. Benigni, J. Rogez, and J. C. Mathieu

Citation: Review of Scientific Instruments 66, 193 (1995); doi: 10.1063/1.1145256

View online: http://dx.doi.org/10.1063/1.1145256

View Table of Contents: http://scitation.aip.org/content/aip/journal/rsi/66/1?ver=pdfcov

Published by the AIP Publishing

\section{Articles you may be interested in}

A steady-state high-temperature method for measuring thermal conductivity of refractory materials

Rev. Sci. Instrum. 84, 054902 (2013); 10.1063/1.4804258

Method for measurement of the thermal diffusivity in solids: Application to metals, semiconductors, and thin materials

J. Appl. Phys. 84, 6327 (1998); 10.1063/1.368957

Transient Thermal Diffusivity Technique for Refractory Solids

J. Appl. Phys. 34, 3550 (1963); 10.1063/1.1729256

Apparatus for the Measurement of the Thermal Diffusivity of Solids at High Temperatures

J. Appl. Phys. 31, 1585 (1960); 10.1063/1.1735897

Several New Methods to Measure the Thermal Diffusivity of Semiconductors

J. Appl. Phys. 31, 612 (1960); 10.1063/1.1735645

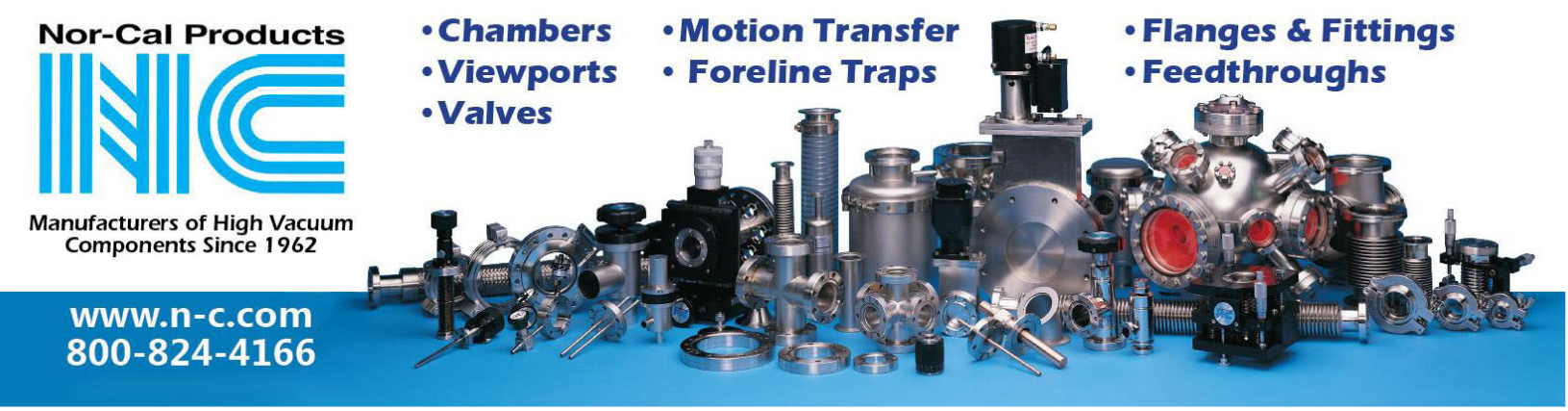




\title{
New apparatus for thermal diffusivity measurements of refractory solid materials by the periodic stationary method
}

\author{
J. Khedari, P. Benigni, J. Rogez, and J. C. Mathieu \\ Centre de Thermodynamique et Microcalorimétrie du C.N.R.S., 26 rue du 141ème R.I.A. 13331 Marseille \\ Cedex 3, France
}

(Received 3 June 1994; accepted for publication 22 September 1994)

\begin{abstract}
An apparatus for the thermal diffusivity measurement of refractory solids at high temperatures $(800-1800 \mathrm{~K})$ by the periodic stationary method is presented. A cylindrical geometry for the sample has been chosen. The radial heat flow through the measurement cell is sine modulated and induces a thermal sine wave over the lateral side of the sample. The thermal diffusivity is determined from the measurement of the phase change and the amplitude ratio between two points of the sample. Thus, the thermal diffusivity can be obtained in two manners. The difference between these results is a criterium for the validity of the measurement. Experimental results on a sample of a cordierite based sintered material (Cecorite 130P) are presented. They show good agreement with the results of the CODATA Task Group on Thermophysical Properties. (C) 1995 American Institute of Physics.
\end{abstract}

\section{INTRODUCTION}

A new high-temperature apparatus has been constructed in order to perform thermal diffusivity measurements of solid samples up to $1800 \mathrm{~K}$. This new device is very close to our high-temperature adiabatic calorimeter which we use in particular for heat capacity measurements. ${ }^{1}$ The same sample can be tested with both apparatuses. The periodic stationary method, first proposed by Angström, ${ }^{2}$ has been chosen with radial heat flow over the cylindrical sample. The principle of this method is simple, but it requires a long time for the measurements.

\section{THE MEASUREMENT METHOD}

In the periodic stationary method, the sample is assumed to be an infinite circular cylinder of radius $b$. A sine temperature wave is transmitted over the lateral face. Accounting for the sample geometry, the main heat transfer equation is

$$
\frac{\partial^{2} T}{\partial r^{2}}+\frac{1}{r} \frac{\partial T}{\partial r}-\frac{1}{K} \frac{\partial T}{\partial t}=0
$$

where $T(r, t)$ is the temperature at the distance $r$ from the axis at time $t$ and $K$ the global thermal diffusivity.

Setting the origin of the phase at $r=b$, the boundary condition can be written as

$$
T(b, t)=T_{i}+\Theta_{b} \cos \omega t,
$$

where $\omega$ is the angular velocity, $T_{i}$ the mean temperature of the measurement, and $\Theta_{b}$ the amplitude of the incident thermal wave.

We can write that, at the center of the sample,

$$
T(0, t)=T_{i}+\theta_{0} \cos \left(\omega t-\Phi_{0}\right),
$$

where $\Phi_{0}$ is the phase of the thermal wave at $r=0$, and to generalize:

$$
T(r, t)=T_{i}+\Theta_{r} \cos \left(\omega t-\Phi_{r}\right) .
$$

The problem is to find the expressions of the amplitude ratio $\Theta_{0} / \Theta_{r}$ and the phase change $\Phi_{0}-\Phi_{r}$. The steady-state solution is written as a periodic complex function of space and time:

$$
T(r, t)=A(r) \exp (i \omega t) .
$$

So the main equation (1) becomes a Bessel equation of zero order:

$$
\frac{\partial^{2} A}{\partial r^{2}}+\frac{1}{r} \frac{\partial A}{\partial r}-i e^{2} A=0, \quad \text { where } e^{2}=\frac{\omega}{K} .
$$

The general solution is taken as

$$
A(r)=C . J_{0}\left(e r i^{3 / 2}\right)+B . K_{0}\left(e r i^{1 / 2}\right),
$$

where $J_{0}$ and $K_{0}$ are zero order Bessel functions. For $r=0$, $K_{0}$ bccomcs infinitc so $B=0$ because $A(r)$ must remain finite. Combining Eq. (2) with Eqs. (3) and (4) yields: ${ }^{3-5}$

$$
T(r, t)=\Theta_{b} \frac{J_{0}\left(e r i^{3 / 2}\right)}{J_{0}\left(e b i^{3 / 2}\right)} \exp (i \omega t),
$$

where $J_{0}$ can be reduced to Kelvin functions ${ }^{6}$ with

$$
J_{0}\left(z i^{3 / 2}\right)=\operatorname{ber}(z)+i . b e i(z) .
$$

Finally, $T$ can be written as

$$
T(r, t)=\Theta_{b} \frac{D \cos \omega t+E \sin \omega t}{F}
$$

with:

$$
\begin{aligned}
D= & b e r(e r) b e r(e b)+b e i(e r) b e i(e b) \\
& +i[\text { bei }(e r) b e r(e b)-b e r(e r) b e i(e b)], \\
E= & b e r(e r) b e i(e b)-b e i(e r) b e r(e b) \\
& +i[\text { ber }(e r) b e r(e b)+b e i(e r) b e i(e b)], \\
F= & b e r^{2}(e b)+b e i^{2}(e b) .
\end{aligned}
$$

The real part of the complex temperature is written as 


$$
T(r, t)=\Theta_{b} \frac{[\operatorname{ber}(e r) b e r(e b)+b e i(e r) b e i(e b)] \cos \omega t+[b e r(e r) b e i(e b)-b e i(e r) b e r(e b)] \sin \omega t}{b e r^{2}(e b)+b e i^{2}(e b)}
$$

Let $d$ be the distance between the inner and the outer thermocouples. The measurements of the amplitude ratio and the phase change between $r=0$ and $r=d$ yield the thermal diffusivity $K$. Experimentally, the amplitude ratio is determined from the measurement of the amplitudes $\Theta_{d}[T(d, t)]$ and $\Theta_{0}[T(0, t)]$.

If the real temperature is written as $T(r, t)=\Theta_{r} \cos \left(\omega t-\Phi_{r}\right)$

$$
\Theta_{r}=\Theta_{b} \frac{\sqrt{[\text { ber }(e r) b e r(e b)+b e i(e r) b e i(e b)]^{2}+[\text { ber }(e r) b e i(e b)-b e i(e r) b e r(e b)]^{2}}}{\operatorname{ber}^{2}(e b)+b e i^{2}(e b)} \text {. }
$$

We can write according to the definition of the Kelvin functions $b e i(0)=0$ and $b e r(0)=1$, and using Eq. (7):

$$
\Theta_{0}=\Theta_{b} \frac{1}{\sqrt{b e r^{2}(e b)+b e i^{2}(e b)}}
$$

and

$$
\Theta_{d}=\Theta_{b} \frac{\sqrt{b e r^{2}(e d)+b e i^{2}(e d)}}{\sqrt{b e r^{2}(e b)+b e i^{2}(e b)}} .
$$

Thus the amplitude ratio $\Theta$ between the center of the sample $r=0$ and $r=d$ is now

$$
\Theta=\frac{\Theta_{0}}{\Theta_{d}}=\frac{1}{\sqrt{b e r^{2}(e d)+b e i^{2}(e d)}} .
$$

The phase change is determined between an extremum of the $T(d, t)$ curve and the corresponding extremum of the $T(0, t)$ curve. Using Eq. (6), the phase is written as

$$
\Phi_{r}=\tan ^{-1}\left(\frac{\text { ber }(e r) b e i(e b)-b e i(e r) b e r(e b)}{\text { ber }(e r) b e r(e b)+b e i(e r) b e i(e b)}\right) .
$$

In the same way, we can write

$$
\Phi_{d}=\tan ^{-1}\left(\frac{\operatorname{ber}(e d) b e i(e b)-b e i(e d) b e r(e b)}{\operatorname{ber}(e d) b e r(e b)+b e i(e d) b e i(e b)}\right)
$$

and

$$
\Phi_{0}=\tan ^{-1}\left(\frac{b e i(e b)}{\operatorname{ber}(e b)}\right) .
$$

With the classic trigonometric formula

$$
\tan (x+y)=\frac{\tan x-\tan y}{1+\tan x \cdot \tan y} .
$$

The phase change $\Phi$ is finally written as

$$
\Phi=\Phi_{0}-\Phi_{d}=\tan ^{-1}\left(\frac{b e i(e d)}{b e r(e d)}\right) \text {. }
$$

The plots $\Phi(u)=\tan ^{-1}[$ bei $(u) /$ ber $(u)]$ and $\Theta(u)$ $=1 / \sqrt{b e r^{2}(u)+b e i^{2}(u)}$ (Fig. 1) give the values of $u$ $=e . d=d \cdot \sqrt{\omega / K}$ for each measurement. Therefore, two values of the diffusivity are obtained, $K_{\Theta}$ from the amplitude ratio and $K_{\Phi}$ from the phase change.

The thermal diffusivity is determined with:

$$
K=\omega \cdot\left(\frac{d}{u}\right)^{2}
$$

where $\omega=2 \pi / p$ and $p$ is the period of the sine wave.

\section{THE DESCRIPTION OF THE MEASUREMENT CELL AND THE GENERAL DISPLAY}

The experimental setup is shown in Fig. 2. The sample is placed inside the cylindrical molybdenum heating shield $\left(H_{a}\right)$. The microfurnace $\left(H_{c}\right)$, the height of which equals that of the sample, provides a heat flow through the shield $\left(H_{a}\right)$. The measurement cell is plunged into the main furnace $(K)$ which controls the mean temperature during the experiment within $\pm 1 \mathrm{~K}$.

The electric circuitry is shown in the block diagram on Fig. 3. The very low-frequency sine wave generator provides the modulation to the microfurnace power supply within $\pm 0.1 \%$. The temperature measurements are performed by means of Pt/Pt-Rh10\% thermocouples $\left(M_{a}, M_{b}\right)$.

The measurement cell hangs from a molybdenum hook $(S)$ (or tungsten for very high-temperature measurements). The sample $(E)$ is a cylinder of $35 \mathrm{~mm}$ diameter and $70 \mathrm{~mm}$ height. The inner thermocouple $\left(M_{a}\right)$ is placed inside an axial hole of $1.7 \mathrm{~mm}$ diameter and $35 \mathrm{~mm}$ depth. The outer thermocouple is placed inside a similar hole $3 \mathrm{~mm}$ from the outer wall of the cylinder. The dimensions of all the elements are determined in order to adjust the time constants which are highly important when a periodic steady state has to be established.

In order to decrease the metallization of the ceramic

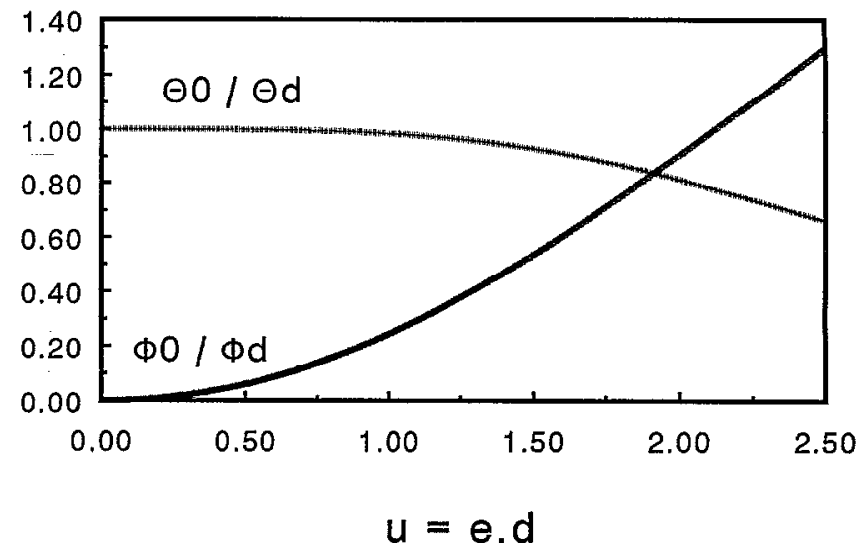

FIG. 1. The phase change and the amplitude ratio in terms of the $u$ parameter. 


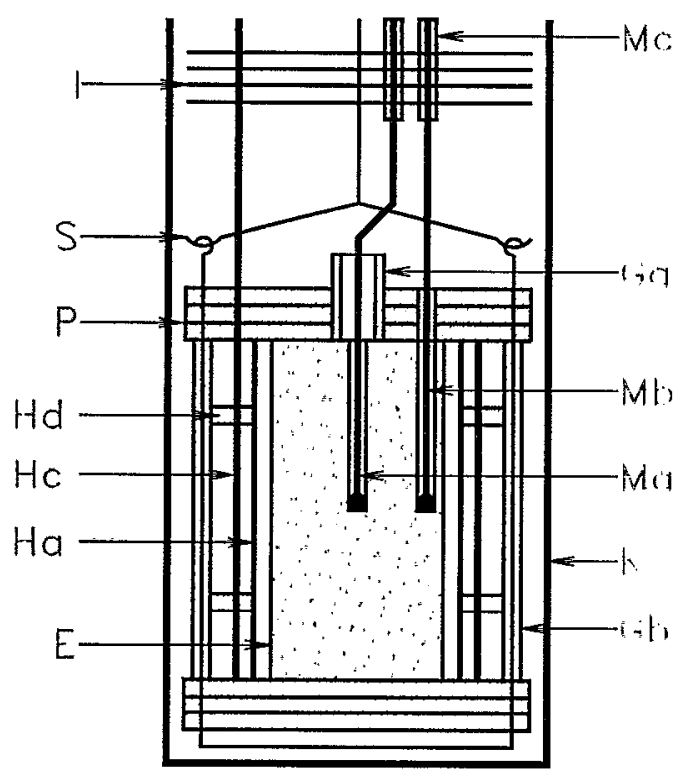

FIG. 2. The measurement cell. E-Cylindrical sample $(\varnothing 35 \mathrm{~mm}$, height 70 $\mathrm{mm}$ ). Ga-Additional alumina insulator of the inner thermocouple (outer $\varnothing$ $10 \mathrm{~mm}$ ). Gb-Alumina insulator of the hook. Ha-Heating shield. Molybdenum cylinder $(\varnothing 45 \mathrm{~mm}$, height $70 \mathrm{~mm}$, thickness $0.15 \mathrm{~mm})$. HcCylindrical microfurnace $(\varnothing 47 \mathrm{~mm}$, height $70 \mathrm{~mm})$. Molybdenum wire $(\varnothing$ $0.8 \mathrm{~mm}$ ). $\mathrm{Hd}$-Alumina rings which strengthen the molybdenum winding. I-Circular shields. Molybdenum plates. K-Main furnace. Molybdenum cylinder ( $\varnothing 60 \mathrm{~mm}$, height $370 \mathrm{~mm}$, wall thickness $0.15 \mathrm{~mm}$ ). Ma-Inner alumina insulated thermocouple (wire $\varnothing 0.3 \mathrm{~mm}$ ). Mb-Outer alumina insulated thermocouple (wire $\varnothing 0.3 \mathrm{~mm}$ ). Mc-Additional alumina insulator of the thermocouples (outer $\varnothing 1.5 \mathrm{~mm}$ ). P-Alumina plates $(\varnothing 55 \mathrm{~mm}$, thickness $5 \mathrm{~mm}$ ). S-Molybdenum or tungsten hook (wire $\varnothing 1 \mathrm{~mm}$ ).

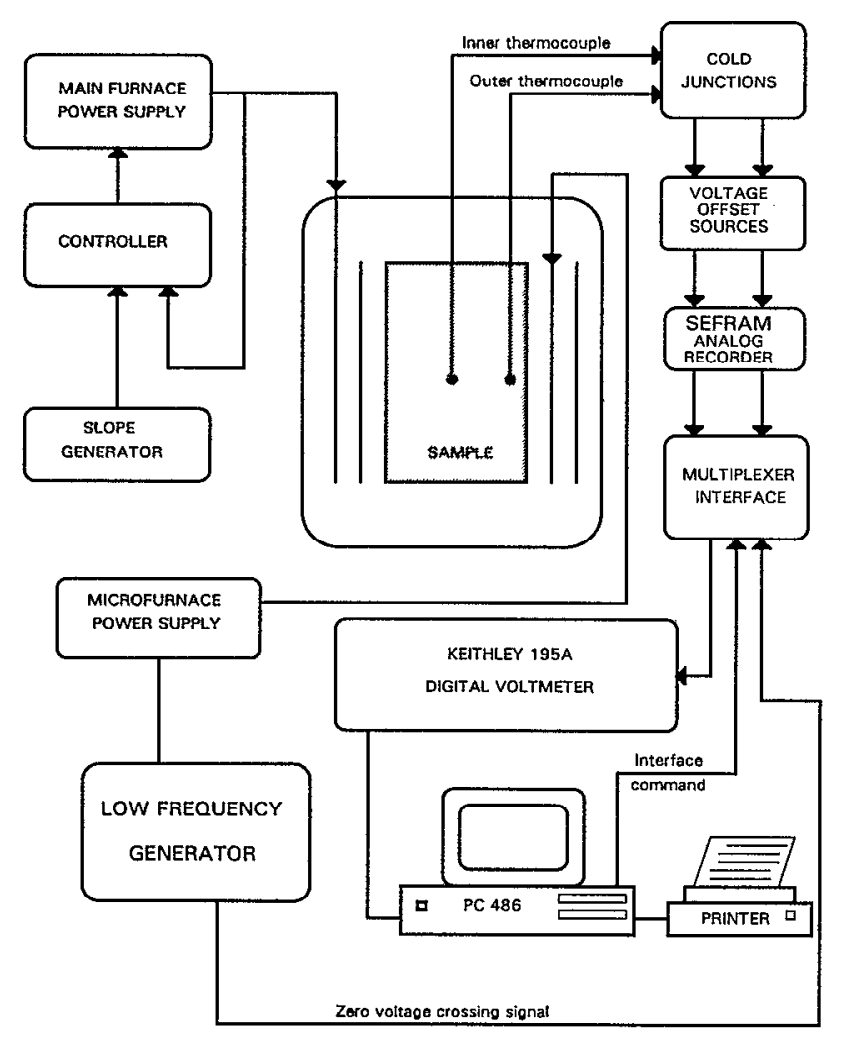

FIG. 3. The block diagram of the electric circuitry. parts at high temperatures, argon is introduced into the main furnace before heating.

\section{THE AUTOMATIC TREATMENT}

The results are treated automatically by a Fourier analysis of the outer and inner temperature recordings. The phase change $\Phi$ and the amplitude ratio $\theta$ are computed from the coefficients of the harmonic analysis. By numerical dichotomy on the plots $\Phi(u)$ and $\Theta(u)$ two values of $u$ are reached and then two values of $K\left(K_{\Phi}\right.$ and $\left.K_{\Theta}\right)$.

As soon as the steady state is established, the thermal signals are sampled during generally 8 periods of the phenomenon. The signals are determined by $(2 N+1)$ discrete values $f_{i}$ on which a Fourier split of $M \leqslant N$ order, as

$$
f(t)=\frac{A_{0}}{2}+\sum_{n=1}^{N}\left[A_{n} \cos (n \omega t)+B_{n} \sin (n \omega t)\right] .
$$

The $(2 N+1)$ coefficients $A_{0}, A_{n}, B_{n}$ are obtained by a Goertzel algorithm. ${ }^{7}$

$$
\begin{aligned}
& A_{0}=\frac{2}{2 N+1} \sum_{i=1}^{2 N+1} f_{i}, \\
& A_{n}=\frac{2}{2 N+1} \sum_{i=1}^{2 N+1} f_{i} \cos \left(\frac{2 \pi i}{2 N+1} n\right), \\
& B_{n}=\frac{2}{2 N+1} \sum_{i=1}^{2 N+1} f_{i} \sin \left(\frac{2 \pi i}{2 N+1} n\right) .
\end{aligned}
$$

For a $n$ periods recording, only the $A_{n}$ and $B_{n}$ coefficients give the phase $\Phi_{n}$ and the amplitude ratio $\theta_{n}$ for each thermogram:

$$
\Theta_{n}=\sqrt{A_{n}^{2}+B_{n}^{2}} \text { and } \Phi_{n}=\tan ^{-1}\left(\frac{B_{n}}{A_{n}}\right) \text {. }
$$

Then, the phase change and the amplitude ratio between the two waves are computed.

$$
\Phi=\Phi_{0}-\Phi_{d} \text { and } \Theta=\frac{\Theta_{0}}{\Theta_{d}} \leqslant 1 .
$$

The automatic treatment allows a better measurement of the phase change, the amplitude ratio, the period and a more precise determination of the $u$ values than a previous manual one. Also, the examination of the $A_{n}$ and $B_{n}$ coefficients of the primary signal gives an idea about the purity of this thermal wave. An harmonic signal can be considered sinely pure when the harmonic distortion ratio (hdr) remains less than $0.1 \%$.

$$
\operatorname{hdr}=\left(\frac{\sum_{i=2}^{N}\left(X_{i \mathrm{rms}}\right)^{2}}{\sum_{i=1}^{N}\left(X_{i \mathrm{rms}}\right)^{2}}\right) \quad \text { with } X_{i \mathrm{rms}}=\frac{\sqrt{A_{i}^{2}+B_{i}^{2}}}{2} .
$$

$X_{i \text { rms }}$ is the root mean square of the $i$ th harmonic, $A_{i}$ and $B_{i}$ are the Fourier analysis coefficients, $N$ the sampling number for a period. ${ }^{8}$

This ratio shows the difference between the inspected wave and a pure sine curve. The determination of the hdr 
depends on the number of sampling points by period. The greater the sampling rate the lower the hdr. However, the determination of the phase change and the amplitude ratio do not depend strongly on this ratio. For the thermal diffusivity computation, the value of the period $p$ has a great influence. This value is more accurately attained by a mean calculation over a recording of several periods. This recording also allows an evaluation of the thermogram shift by applying a least squares method ${ }^{9}$ upon the $A_{0}$ coefficients of the Fourier analysis for each period. Eventually, before the harmonic treatment, the thermograms are corrected for this shift.

Finally the error lies uniquely in keeping only $n$ Fourier analysis coefficients, a finite sampling rate and in the determination of the period.

\section{THE STUDY OF THE EXPERIMENTAL ERRORS}

The initial test material was a $99.7 \%$ purity $\alpha$-alumina the porosity of which is about $5 \%$. The outer diameter of the cylinder is smaller than that used in further analysis $(\varnothing$ $=24.6 \mathrm{~mm}$ ). The literature data for alumina has a great deal of scatter. So, it is difficult to compare the preliminary results obtained between 800 and $1800 \mathrm{~K}$ in order to calibrate our apparatus. These values are not reported here because the sample has not been well characterized and its size is not at the optimum required for precise measurements.

Yet a systematic study with the frequency of the incident wave has shown that the $K_{\Phi}$ and $K_{\Theta}$ measurements depends on $\omega$. This behavior seems to have been already observed by other authors ${ }^{10,11}$ who have previously used this method but no explanation at present exists. This dependency is different for $K_{\Phi}$ and $K_{\Theta}$. So, there is a gap between both values at a single $\omega$. This observation has often been hidden in the literature because either the phase change or the amplitude ratio are separately measured.

In order to clarify this situation, the grounds for error have been studied in detail. We shall distinguish the random errors from the systematic errors.

(a) The random errors are mainly due to the experimental setup and the method of automatic treatment which yield the phase change, the amplitude ratio, and the period. ${ }^{12}$ They also include the radius measurement. They can be estimated at about $\pm 2 \%$.

(b) The systematic errors are due to the discrepancy between the theoretical principle of the method and its experimental carrying out. Is our experimental setup in agreement with the infinite cylinder hypothesis? A number of tests have been implemented and the results are discussed below.

(i) The uniformity of the incident heat flow has been tested using two special setups with three thermocouples welded at various places over the lateral surface of the cylinder. In the first one, the sensors were regularly placed in the half height plan of the sample. In the second one, the thermocouples were welded on the same meridian at half height and at $\pm 10 \mathrm{~mm}$ of this half height. Within the error margin, it has been verified that the incident heat flow is radially and longitudinally uniform over a reasonable area around the measurement points. The thermal sine wave is considered pure enough because the discrepancy between the hdr of the electronic wave provided by the generator and the outer temperature signal hdr is less than $1 \%$. When crossing the molybdenum heat shield and the argon atmosphere, the thermal sine shaped signal is not perturbed.

(ii) In a previous setup, no alumina shields were placed on or under the sample. An axial heat flow might disturb the radial heat flow which is the only one described in the ground equation (1). So the influence of this axial heat flow has been studied. ${ }^{13,14}$ Comparison of the solutions in the finite and infinite cylinder with periodic boundary conditions have shown that the difference between both solutions depend on the radius and the frequency but did not exceed $1 \%$ for the measuring points.

(iii) The inner thermocouple is introduced in a cylindrical $2 \epsilon$ diameter hole. The difference $\Phi(r=0)-\Phi(r=\epsilon)$ depends on the frequency. It remains less than $1 \% .^{14}$

(iv) The heat leak through the inner thermocouple has been studied. ${ }^{14}$ The consequent error depends also on the frequency but remains less than $2 \%$.

(v) What do the thermocouples data represent? The thermocouple indication is not exactly the temperature at radius $r$ and time $t \cdot{ }^{15,16}$ The thermal field around the measuring points is locally disturbed by the sensor. The measure is indeed an intermediate value between the extrema of the disturbed zone. During a periodic steady state, this remark becomes much more important. In order to overcome this drawback the temperature has been averaged by integration over a cylindrical zone surrounding the thermocouples welding. It is difficult to determine correctly the limits of this integration zone. Some authors proposed for example ten times the thermocouple diameter. ${ }^{15} \mathrm{~A}$ numerical integration of the thermal field, around the inner and outer thermo-

TABLE I. Diffusivity vs period at different temperatures. Each value is an average over at least three measurements. The order of magnitude of the deviation can be seen on the figures.

\begin{tabular}{cccccccccccc}
\hline \hline & \multicolumn{10}{c}{ Period in $s$} \\
\cline { 2 - 12 } Temperature & \multicolumn{10}{c}{146} & \multicolumn{10}{c}{252} & \multicolumn{2}{c}{333} & \multicolumn{2}{c}{492} & \\
\hline K & a & h & a & h & a & b & a & b & a & b \\
867 & 7.19 & 7.63 & 7.23 & 7.50 & 7.29 & 7.48 & 7.36 & 7.49 & 7.20 & 7.51 \\
1266 & 6.99 & 7.07 & 7.16 & 7.10 & 7.26 & 7.12 & 7.82 & 7.31 & 7.93 & 7.42 \\
$881^{\mathrm{c}}$ & 5.95 & 7.18 & 5.52 & 7.15 & 5.40 & 6.63 & 5.19 & 6.35 & 4.96 & 6.19 \\
$1234^{\mathrm{c}}$ & 5.94 & 6.57 & 5.96 & 6.48 & 5.89 & 6.44 & 5.81 & 6.33 & 5.75 & 6.30 \\
\hline
\end{tabular}

$K_{\Theta} \cdot 10^{3}$ in $\mathrm{cm}^{2} \mathrm{~s}^{-1}$

$K_{\Phi} \cdot 10^{3}$ in $\mathrm{cm}^{2} \mathrm{~s}^{-1}$.

'Heat Treated. 


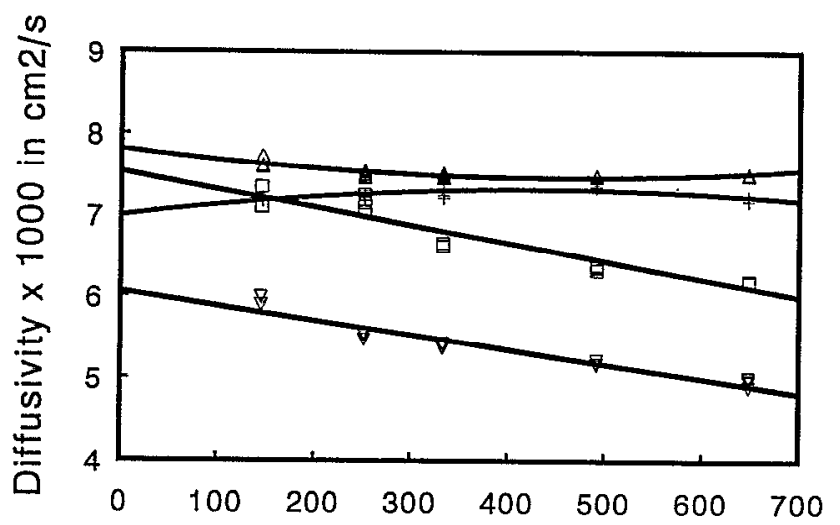

Period in $\mathrm{s}$ ists. In order to test our apparatus, the best material available seems to be the Cecorite 130P. This material is a cordicrite based sintered ceramic provided by the Lafarge Company, France. The chemical analysis is $\mathrm{Al}_{2} \mathrm{O}_{3} 34.5-\mathrm{MgO} 7.5-\mathrm{SiO}_{2}$ 58. The Task Group on Thermophysical Properties ${ }^{18}$ has chosen this material as a standard reference candidate in a CODATA measurement program focused on thermal properties of low-conductivity materials.

The thermal diffusivity has been measured by nine different experimentalists using five different methods. For better homogeneity, all the samples were cut from the same Cecorite plate. The results show a $\pm 10 \%$ scattering with respect to a fourth order polynomial fit upon all the measurements. ${ }^{18}$

Our sample is also from the original Cecorite plate.

FG. 4. Cecorite $130 \mathrm{P}, T=867 \mathrm{~K} .+: K_{\Theta} \triangle: K_{\Phi} T=881 \mathrm{~K}$, heat treated. $\nabla$ : $K_{\boldsymbol{\theta}} \square: K_{\Phi}$

couples, by a Simpson method ${ }^{17}$ has been performed using various integration zone radii up to $3 \mathrm{~mm}$. A greater radius should mean that the outer thermocouple is sensitive to the gaseous atmosphere which surrounds the sample. According to the isoamplitudes and isophases distribution in the sample ${ }^{13}$ the integration has shown a greater influence upon the outer thermocouple than upon the inner one. For the greatest radius expected $(3 \mathrm{~mm})$ the integration yields an increase in $K_{\Phi}$ and a decrease in $K_{\circledast}$ by $1 \%$. This effect decreases with the period and becomes nearly zero for great periods.

The results of these studies permit one to understand and reduce some systematic errors but not to explain the dependency of the measurements on the frequency and the gap between the $K_{\Phi}$ and $K_{\Theta}$ measurements.

\section{THE CALIBRATION OF THE APPARATUS}

\section{A. The choice of a standard material}

In the field of high-temperature technology, no standard reference material for thermal diffusivity measurements ex-

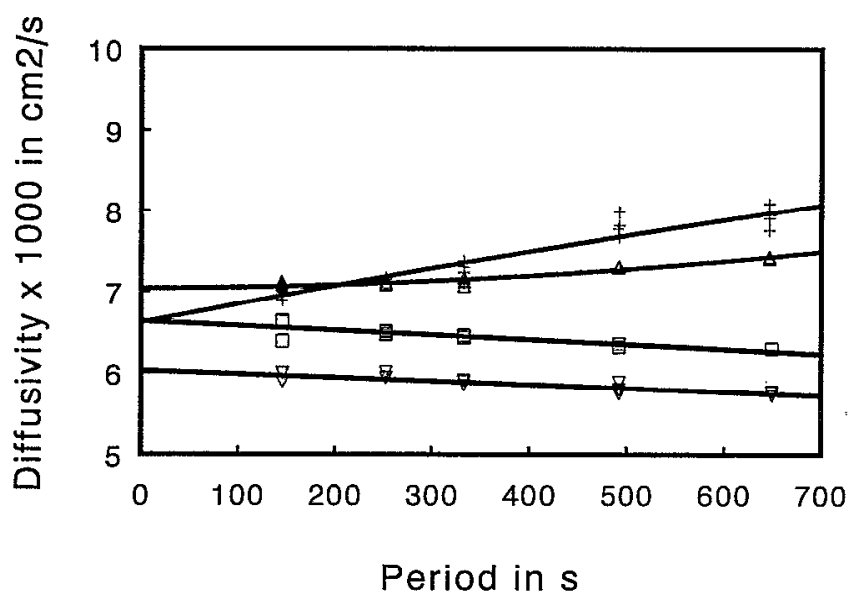

FIG. 5. Cecorite $130 \mathrm{P}, T=1266 \mathrm{~K} .+: K_{\circledast} \triangle: K_{\Phi} T=1234 \mathrm{~K}$, heat treated. $\nabla: K_{\Theta} \square: K_{\Phi}$

\section{B. The experimental results}

A first set of measurements has been performed on the "as-received" sample. The data obtained at 867 and $1266 \mathrm{~K}$ (Table I) are plotted versus the period on Figs. 4 and 5. As with alumina, the dependency on the frequency is observed. In the $1266 \mathrm{~K}$ plot, the two values $K_{\Phi}$ and $K_{\Theta}$ are equal around the $200 \mathrm{~s}$ period.

As is commonly done, a mean value for diffusivity is calculated including all the results obtained indistinguishly from the phase change and the amplitude ratio. At each temperature, the average value is about $30 \%$ greater than that issued from the literature fit.

Some participants of the CODATA program have observed such a discrepancy in the first measurements. Connett noticed a dependency of his measurements with the thermal history of the sample. Maglic noticed an optical stabilization of his sample after the first heating.

In order to increase the structural and chemical equilibrium of the sample, the following thermal treatment has been applied: A three days $1523 \mathrm{~K}$ heating followed by a mean cooling at $200 \mathrm{~K}$ per hour down to room temperature.

A second set of measurements has been performed at $881 \mathrm{~K}$ (Table I and Fig. 4) and $1184 \mathrm{~K}$ (Table I and Fig. 5). The results are well fitted by straight lines, the slopes of which are small and nearly equal. The $K_{\Phi}-K_{\Theta}$ difference remains constant over the whole period range. If we hold only the average $K_{\Theta}$ value as it is commonly done, a good agreement is found with the literature data, within the error margin.

At this stage of the study, we can consider that it is possible to measure a global thermal diffusivity coefficient in the same manner as other apparatuses. However, experimental results have shown a dependency of the measurements upon the period of the thermal wave and a gap between the diffusivities obtained from the phase change and the amplitude ratio.

Now, it is thought that the main hypothesis expressed in Eq. (1) is only nearly exact. The heat transfer is not well represented by a single conduction coefficient and a single diffusion equation but at least by two coefficients representative of pure conduction and radiation. The system of non- 
linear integrodifferential equations describing the heat transfer is much more complex than Eq. (1). The solution must be quite but not exactly the same as the present one. Attempts in this direction are now investigated.

${ }^{1}$ J. Rogez and J. Le Coze, Rev. Phys. Appl. 15, 341 (1980).

${ }^{2}$ A. J. Angström, Ann. Phys. Chem. 12, 513 (1861).

${ }^{3}$ H. S. Carslaw and J. C. Jaeger, Conduction of Heat in Solids (Oxford University Press, New York, 1959).

${ }^{4}$ M. N. Ozizik, Heat Conduction (Wiley, New York, 1980).

${ }^{5}$ A. B. De Vriendt, La Transmission de la Chaleur (Gaëtan Morin, Paris, 1982).

${ }^{6}$ A. B. Abramowitz and I. A. Stegun, Handbook of Mathematical Functions with Formulas (Dover, New York, 1970).

${ }^{7}$ G. Goertzel, A. Ralston, and H. S. Wilf, Méthodes Mathématiques pour Calculateur Arithmétiques (Dunod, Paris, 1965), pp. 425-431.
${ }^{8}$ E. Fromy, Mesures en Radiotechnique 241-254 (Dunod, Paris, 1958), pp. 241-254.

${ }^{9} \mathrm{~J}$. Legras, Précis d'Analyse Numérique, (Dunod, Paris, 1963), pp. 22-25.

${ }^{10} \mathrm{~J}$. C. Marechal and J. M. Devisme, Annales de l'institut Technique du Bâtiment et des Travaux Public 357, 82 (1978).

${ }^{11}$ R. D. Cowan, J. Appl. Phys. 32, 1363 (1961).

${ }^{12}$ J. Khedari, Thèse, Université de Provence, Marseille, France, 1990).

${ }^{13}$ J. Khedari, P. Benigni, J. Rogez, and J. C. Mathieu, Proc. R. Soc. 438, 319 (1992).

${ }^{14}$ H. Rouault, J. Khedari, C. Arzoumanian, and J. Rogez, High Temp. High Press. 19, 357 (1987).

${ }^{15} \mathrm{~J}$. P. Bardon, Aspect Thermocinétique des Erreurs de Mesure de Température par Contact (Inst. de Phys., Nantes, France, 1970), pp. 65-86.

${ }^{16} \mathrm{~J}$. Rogez and J. Le Coze, Mesure des températures, Techniques de l'Ingénieur, Traité Mesures et Contrôle R 2515 1-1992.

${ }^{17} \mathrm{H}$. Haut, Mathématiques et statistiques, programmes en BASIC, Editions du P.S.I., Lagny-sur-Marne, France (1981).

${ }^{18}$ F. Cabannes and M. L. Minges, High Temp. High Press. 21, 69 (1989). 